\title{
The Formation of First Generation Stars and Globular Clusters in Protogalactic Clouds
}

S. Murray and D. N. C. Lin

This article was presented to:

IAU Symposium \#187

Kyoto, Japan

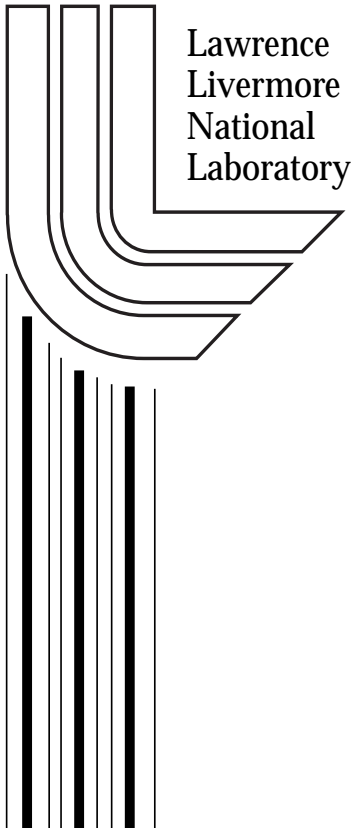

August 1997 


\section{DISCLAIMER}

This document was prepared as an account of work sponsored by an agency of the United States Government. Neither the United States Government nor the University of California nor any of their employees, makes any warranty, express or implied, or assumes any legal liability or responsibility for the accuracy, completeness, or usefulness of any information, apparatus, product, or process disclosed, or represents that its use would not infringe privately owned rights. Reference herein to any specific commercial product, process, or service by trade name, trademark, manufacturer, or otherwise, does not necessarily constitute or imply its endorsement, recommendation, or favoring by the United States Government or the University of California. The views and opinions of authors expressed herein do not necessarily state or reflect those of the United States Government or the University of California, and shall not be used for advertising or product endorsement purposes.

This is a preprint of a paper intended for publication in a journal or proceedings. Since changes may be made before publication, this preprint is made available with the understanding that it will not be cited or reproduced without the permission of the author. 


\title{
THE FORMATION OF FIRST GENERATION STARS AND GLOBULAR CLUSTERS IN PROTOGALACTIC CLOUDS
}

\author{
D.N.C. LIN \\ Lick Observatory, Univ. of California, Santa Cruz, CA 95064 \\ AND \\ S.D. MURRAY \\ Lawrence Livermore Nat'l Lab., L-23, Livermore, CA 94550
}

\begin{abstract}
Within collapsing protogalaxies, thermal instability leads to the formation of a population of cool fragments which are confined by the pressure of a residual hot background medium. The critical mass required for the cold clouds to become gravitationally unstable and to form stars is determined by both their internal temperature and external pressure. Massive first generation stars form in primordial clouds with sufficient column density to shield themselves from external UV photons emitted by nearby massive stars or AGNs. Less massive photoionized clouds gain mass as they undergo cohesive collisions with each other, and lose mass due to ram pressure stripping by the residual halo gas. Collisions may also trigger thermal instability and fragmentation into cloudlets. While most cloudlets have substellar masses, the largest become self-gravitating and collapse to form protostellar cores without further fragmentation. The initial stellar mass function is established as these cores capture additional residual cloudlets. Energy dissipation from the mergers ensures that the cluster remains bound in the limit of low star formation efficiency. Dissipation also promotes the formation and retention of the most massive stars in the cluster center. On the scale of the protogalactic clouds, the formation of massive stars generates intense UV radiation which photoionizes gas and quenches star formation in nearby regions. As gas density accumulates in the center of the the galactic potential, the self regulated star formation rate increases. At the location where most of the residual gas can be converted into stars on its internal dynamical timescale, a galaxy attains its asymptotic kinematic structure such as exponential profiles, Tully-Fisher, and Faber-Jackson laws.
\end{abstract}




\section{Fragmentation of Protogalactic Clouds}

During the collapse of a protogalactic cloud (PGC), density in homogeneities and velocity variations lead to shocks which heat the gas to the virial temperature $\left(\sim 10^{6-7} \mathrm{~K}\right)$ of the galactic halo (Binney 1977; Rees \& Ostriker 1977; White \& Rees 1978). In order for a PGC to collapse, its cooling time scale, $\tau_{c}$, must be shorter than the dynamical time scale, $\tau_{d}$, on which it can contract. For PGC's with masses comparable to the Galaxy, this condition is satisfied when their length scale $D<100 \mathrm{kpc}$ (Blumenthal et al. 1984).

The emergence of small scale structure requires that either the growth rate or the limiting amplitude of any perturbations be decreasing functions of their length scale. In a non rotating, cold, homogeneous cloud, gravitational instability alone cannot induce fragmentation, because its growth rate is essentially independent of length scale. In the limit that $\tau_{c}<\tau_{d}$, thermal instability can lead to the rapid growth of perturbations from infinitesimal to nonlinear amplitudes (Field 1965). At the virial temperature of the PGC, the dominant cooling mechanisms are bremsstrahlung and recombination processes (Dalgarno \& McCray 1972) for which $\tau_{c}$ increases with temperature. In this case, the temperature difference between cooler perturbed regions and the background is amplified leading to the formation of a two phase medium.

Although thermal instability proceeds more rapidly than the collapse of the cloud, its growth rate is essentially independent of length scale, because it is determined by the local cooling rate. Nonetheless, the growth of over dense regions in a thermally unstable cloud is limited by the transition from isobaric to isochoric evolution, and the limiting amplitude is a decreasing function of the length scale (Burkert \& Lin in preparation). Across the interface between the perturbed region and the background, differential cooling leads to a pressure gradient which induces gas flow from the hot background towards the cooler perturbed regions. The density enhancement in the cooler region reduces $\tau_{c} / \tau_{d}$ in the cooler gas, and reduces $\tau_{c}$ in the cooler gas further below that in the background. The pressure-driven acceleration of the interface also induces the onset of Rayleigh-Taylor instability. The growth rate of Rayleigh-Taylor instability increases for small wavelengths, $\lambda$. The growth rate of the small $\lambda$ perturbations, however, decrease when they become nonlinear, ie. when their amplitudes $a \approx \lambda$, and later evolution is dominated by perturbations with larger wavelengths.

The above discussions indicate that although thermal instability can lead to runaway growth of perturbations, it does not lead to their breakup to small length scales. We now examine the processes which can lead to the fragmentation of the cold regions.

Once the final $T$ is attained, pressure balance is re-established on all 
scales and the PGC becomes a two-phase medium with density $(n)$ contrast inversely proportional to the temperature $(T)$ ratio. The residual halo gas (RHG) in the background remains at the virial temperature, with a density such that its thermal energy is lost through both radiative cooling and thermal conduction between it and the cold clouds (McKee \& Cowie 1977). Due to buoyancy effects, the clouds fall toward the center of the galactic potential and dissipate its gravitational to heat the RHG. At a galactocentric radius $D \sim 10 \mathrm{kpc}$, an energy balance is attained with $n T \sim 10^{3-4}$ (Lin \& Murray in preparation).

The cooling efficiencies of bremsstrahlung, recombination, and atomic hydrogen emission decrease rapidly below $\sim 10^{4} \mathrm{~K}$. In metal-free gas, however, non-equilibrium recombination leads to the formation of a small amount of $\mathrm{H}^{-}$ions which combine with neutral $\mathrm{H}$ to form $\mathrm{H}_{2}$. Emission by $H_{2}$ is capable of reducing the gas temperature, $T$ to $\sim 10^{2} \mathrm{~K}$ (Murray \& Lin 1990). In metal-enriched clouds, emission by metal ions, such as CII, molecules such as $\mathrm{CO}$, and grains provide additional cooling to lower equilibrium temperatures. Once the final temperature is attained, pressure balance is re-established on all scales.

The clouds' infall motion is affected by both their coagulation and RHG's drag (Lin \& Yorke in preparation). The motion of the clouds through the RHG also leads to mass loss due to Kelvin-Helmholtz instability (Murray et al. 1993). Since the growth time scale of Kelvin Helmholtz instability increases with the perturbation wavelength, it leads to fragmentation. The break down of the clouds increases their collective area filling factor and so increases their collision frequency. A balance between disruption and coagulation establishes an equilibrium size distribution.

\section{Formation of Protocluster Clouds}

A lower limit on their size distribution is set by the clouds' evaporation due to conduction from the hot RHG. At higher masses, the central density of the clouds increases with mass. Above a critical mass, $M_{c}$, thermal pressure can no longer support the weight of the envelope (Bonner 1956), and the clouds undergo inside-out collapse (Shu 1977). The inside-out collapse is stable and does not lead to fragmentation without any further unstable cooling. Contrary to the opacity-limited fragmentation scenario (Hoyle 1953; Low \& Lynden-Bell 1976), $M_{c}$ represents the minimum mass for isothermal collapsing clouds (Tsai, in preparation).

In primordial gas, $\mathrm{H}_{2}$ emission leads to $T \sim 10^{2} \mathrm{~K}$, which corresponds to $M_{c} \sim 10^{2} M_{\odot}$, and the formation of massive stars. A population of $\sim 10^{4} \mathrm{O} 5$

stars produce adequate UV radiation to photoionize the entire PGC out to $100 \mathrm{kpc}$, which leads to $T \sim 10^{4} \mathrm{~K}$ and $M_{c} \sim 10^{6} M_{\odot}$. Since $M_{c}$ is 
comparable to the mass of globular clusters, we refer these warm, massive clouds as protocluster clouds (PCC's). Clouds with $M<M_{c}$ are stable, and star formation is quenched. As some of the massive stars evolve off the main sequence, the UV flux diminishes, cooling again leads to $T \sim 10^{2} \mathrm{~K}$ in sheltered regions, and spontaneous star formation resumes. This selfregulated star formation scenario has three implications: 1) Stars formed in a metal-poor environment are massive and short-lived, consistent with their rarity today. 2) The elemental abundance distribution is produced by type II supernovae, consistent with that observed among stars with $[\mathrm{Fe} / \mathrm{H}]<-1$ (Wheeler et al. 1989). 3) The self-regulated star formation rate naturally yields $[\mathrm{Fe} / \mathrm{H}] \sim 0.1$ on the collapse time scale of the $\mathrm{PGC}, \tau_{d} \sim 1 \mathrm{Gyr}$, consistent with the halo stars.

We can estimate $n T$ of the PCC's from the values of $n$ and the velocity dispersion $(\sigma)$ of Galactic globular clusters, averaged over their half-mass radius $\left(r_{h}\right)$. These quantities do not change during the post formation evolution. The clouds' radius prior to star formation is probably $>2 r_{h}$ but smaller than their tidal radii (several $r_{h}$ ), such that their initial density may be 1-3 orders of magnitude smaller than the average cluster density at $r_{h}$ today. From $\sigma$ we estimate $T \sim 10^{4} \mathrm{~K}$, comparable to that expected if they were photoionized. Since both the magnitude $\left(\sim 10^{2-5}\right)$, and the dependence on the Galactic distance $\left(\propto D^{-3}\right)$ inferred for $n T$ are consistent with those expected for the RHG (Murray \& Lin 1992), we postulate that PCC's with mass $<M_{c}$ are confined by the RHG's pressure.

From these results, and the cluster metallicities, we can also estimate the cooling time scale $\left(\tau_{c c}\right)$ and dynamical time scale $\left(\tau_{d c}\right)$, of the PCC's. The ratio $\tau_{c c} / \tau_{c d}$ increases from $\sim 10^{-4}$ near the Galactic bulge to $\sim 1$ at $\sim 100 \mathrm{kpc}$. In most PCC's $\tau_{c c}<<\tau_{c d}$ and thermal equilibrium is only possible in the presence of external UV photons with a flux comparable to that of a single O5 star at a distance comparable to the PCC's size ( typically a few pc). This flux is also comparable to that required for self regulated star formation in the halo.

\section{Induced Star Formation in PCC's}

A constraint on the duration of the star formation epoch of a PCC can be imposed from the metal homogeneity (primarily in $[\mathrm{Fe} / \mathrm{H}]$ ) among stars within individual clusters. In $\mathrm{M} 92,[\mathrm{Fe} / \mathrm{H}]=-2.2$ and $\Delta[\mathrm{Fe} / \mathrm{H}]<0.1$ among the cluster stars (Stetson \& Harris 1988). The total metal content in M92 $\left(\sim 30 M_{\odot}\right)$ can be produced by 2 or 3 supernovae. If star formation in M92 occurred over a timescale of more than a few $10^{6}$ years, supernovae would increase both $[\mathrm{Fe} / \mathrm{H}]$ and $\Delta[\mathrm{Fe} / \mathrm{H}]$ to values much larger than observed.

The small $\Delta[\mathrm{Fe} / \mathrm{H}]$ implies that PCC's are enriched and well-mixed 
prior to a rapid burst of star formation. The most efficient mixing process within the PCC's is large scale turbulence (Murray \& Lin 1990), which may be excited by Kelvin-Helmholtz instability at the interface with the RHG. Since shock dissipation damps supersonic motion efficiently, the mixing time scale is typically a few $\tau_{d c} \gg \tau_{c c}$. External heating by nearby massive stars is then required to maintain a stable thermal equilibrium for the PCC's. Such an equilibrium is only stable, however, if the PCC's are not strongly self-gravitating.

In PCC's with marginal self-gravity, mass loss due to ram pressure stripping by RHG is stabilized. Mass loss may also be compensated by coagulation in a collisional equilibrium. Collisions also introduce perturbations which, with a sufficiently large amplitude, could increase the cooling efficiency, leading to star formation (Murray \& Lin 1989). In isolated, but relatively massive PCC's, where self-gravity is important, external heating can no longer stabilize thermal instability (Murray \& Lin 1992), and runaway cooling leads to $T<100 \mathrm{~K}$. The corresponding $n$ enhancement would increase the recombination rate and opacity. With sufficiently large column density, self-shielding may also act to prevent the heating of the PCC's interior. All of these effects indicate that there is a small range of mass over which the PCC's become thermally unstable and begin to collapse.

While most clusters are members of the spheroidal population, the average value of $D$ is smaller than that of the Sun. Dissipative collisions between PCC's reduce their specific energy to values comparable to disk stars, much below that of the PGC. Collisions between two PCC's also lead to the separation of gas from dark matter and older stars which may have formed within them and contributed to their prior enrichment.

The onset of thermal instability, and the subsequent Rayleigh-Taylor and Kelvin-Helmholtz instabilities caused from the resulting collapse of the PCC's induce them to fragment into a population of small cloudlets analogous to the formation of the PCC's within a PGC. The small cloudlets are pressure confined by the warm $\left(10^{4} \mathrm{~K}\right)$ residual gas (WRG) which is, in turn, in pressure balance with the RHG. Due to their collective gravity, and their buoyancy in the WRG, the cloudlets collapses towards the center of PCC. During their infall, the cloudlets lose mass through ram pressure stripping and gain mass through coagulation. Cloudlets with masses $>M_{c}$ collapse to form protostellar cores. Since no additional fragmentation is expected, the minimum stellar mass is $\sim M_{c} \propto T^{2}$.

We cited above circumstantial evidence that the timescale for star formation in clusters is less than a few million years. On such a timescale, cloudlets with adequate metallicity $([\mathrm{Fe} / \mathrm{H}]>-2.5)$ can cool to $10 \mathrm{~K}$ and form low mass stars before the emergence of massive stars which would emit intense UV radiation, photoionize cloudlets, and quench star formation. 
Since their cooling timescale is $>10^{6} \mathrm{yr}$, extremely metal poor cloudlets $([\mathrm{Fe} / \mathrm{H}]<-2.5)$ attain a minimum temperature $>10 \mathrm{~K}$ before being photoionized. Thus, metal poor stars tend to be relatively massive and short lived accounting for their rarity today (Hellsten \& Lin 1998).

Numerical simulations of the coagulation of cloudlets in PCC's (Murray \& Lin 1996) show that initially the geometric cross section determines the collisional frequency between uncollapsed cloudlets, leading to a relatively flat mass distribution. After the cloudlets acquire sufficient mass to form protostellar cores, they continue to acquire additional mass as they merge with residual cloudlets. The increasing mass and compact sizes of the cores, causes their capture rate of residual cloudlets to be strongly affected by gravitational focusing. As a result, their growth timescale decreases with mass. A few massive cores rapidly grow prior to any significant mass increase among most other cores, leading to a steep initial mass function (IMF) for the cores. The cloudlet-core collisions also lead to the dissipation of their relative motion, while global instabilities increase their velocity dispersion (Aarseth et al. 1988). In the final stage of the PCC's collapse, the velocity dispersion increases rapidly, and the collisional frequency is once again determined by the geometrical cross section of the cloudlets, leading to a flattening of the IMF during the collapse. The final slope of the IMF is determined by whether or not most cloudlets have undergone collisions to form protostellar cores before gravitational focusing ceases to be important.

The growth of the most massive cores is terminated when their UV emission heats and ionizes nearby cloudlets. The most massive stars require many dissipative mergers, and so are preferentially formed and tend to remain in the cluster center. This expectation is in contrast to the implication of opacity-limited fragmentation scenario, in which the Jean's criterion suggests that the high mass stars are preferentially formed in low density environments.

After the first generation of stars are formed, the residual gas must be removed from the clusters to avoid self-contamination. Extrapolating from the present stellar population, a Salpeter IMF would imply the existence of tens of massive stars within a typical globular cluster. The UV flux from these massive stars is sufficiently intense to photoionize the residual gas throughout a PCC (Tenorio-Tagle et al 1986). In the shallow potential of PCC's, the resultant internal heating leads to the formation of an expanding ionization wave which efficiently clears out the residual gas within a few $10^{6}$ yr (Noriega-Crespo et al. 1989).

Unless more than half of the gas in PCC's is converted into stars, the removal of the gas on a timescale comparable to the dynamical timescale rapidly reduces the depth of the cluster's potential. In most cases, the disposal of residual gas would then lead to the disruption of the cluster (Lada 
al. 1984). If, however, young stars form through the coagulation of low mass cloudlets, energy dissipation resulting from the mergers leads to the resulting star clusters having radii much smaller than the original PCC's. It is therefore more likely that the newly formed clusters will remain gravitationally bound even in the limit of inefficient star formation. Numerical simulations show up to an order of magnitude reduction in the half mass radius of the star cluster formed through coagulation of a system of collapsing cloudlets (Murray \& Lin 1996).

\section{Self-Regulated Star Formation in Collapsing Protogalactic Clouds}

Various models of star formation during the collapse of a PGC have been constructed in an attempt to match the galactic stellar distributions and chemical en richment history with observational data (Larson 1974). The results of these investigations show that the observations a re best simulated with a star formation law in which the star formation rate $\propto n^{2}$, This empirical prescription is essentially ad hoc and its direct comparison with the observations of current start formation rates in the Galactic disk (Schmidt 1956) may be inappropriate, since the physical conditions in PGCs were probably very different from those present day.

Based on the above considerations, the collapse of a PGC may, in principle, be accompanied by an epoch of efficient star formation in which a large fraction of the PGC is turned into stars. The outcome of such an event would be the formation of galaxies with radii which are much larger than typically observed Holmberg radii. One possible mechanism for suppressing star formation is through external heating due to background UV radiation sufficient to photodissociate $\mathrm{H}_{2}$ molecules, as discussed above in the context of globular clusters.

At large galactic distance $(D>30 \mathrm{kpc}), n<10^{-1}$ for clouds with masses $<M_{c}$ and $T>10^{4}$ because $n T<10^{2-3}$ in the RHG. The cooling rate cannot exceed the rate of photoionization and dissociation resulting from the UV flux of external AGNs. Inside $30 \mathrm{kpc}$, however, adequate UV radiation can only be provided by massive stars formed within the PGC (Lin \& Murray 1992). When the population of massive stars reaches a critical number, they can photoionize and dissociate the entire PGC. Subsequent star formation is suppressed until the massive stars evolve off the main sequence. Both the internal density and the filling factor of the clouds are decreasing functions of $D$. Thus, the recombination rate, the critical UV flux, star formation rate, and heavy element yield all decrease at larger $D$. At some small radii, even with all the residual gas converted into stars on a dynamical timescale, there is insufficient number of massive stars to provide sufficient UV radiation to quench star formation. At that point, 
efficient star formation ensues. The dissipational gaseous collapse at larger $D$ becomes, instead, dissipationless stellar collapse, and the protogalaxy become a bona fide galaxy. The continuous formation of stars leads to a luminosity $\sim 10^{43-44} \operatorname{ergs~s}^{-1}$ and the gas is enriched to $[\mathrm{Fe} / \mathrm{H}]>-2$. Dissipation during the collapse implies that stars will retain a memory of the point at which they formed, leading to the formation of steep metallicity gradients. After virialization, the final radii are comparable to the typical scale lengths observed in galaxies. In the absence of rotation, the mass dependence of the critical radius leads naturally to the Faber-Jackson relation of elliptical galaxies (Lin \& Murray 1992).

Similarly, in disk galaxies, rapid star formation is prevented unless the surface density exceeds a critical value. This condition cannot be reached beyond a radius at which the disk becomes gravitationally unstable before the onset of rapid star formation. This transition radius is comparable to the scale length of exponential disks and its dependence on the galactic mass is similar to that of the Tully-Fisher law. At larger $D$, star formation is self regulated and inefficient, resulting in the observed exponential profiles (Lin \& Pringle 1987).

\section{Comparison With Present-Day Cluster and Star Formation}

In the construction of the above comprehensive model for the formation of first generation stars and stellar clusters, we used the observed dynamical and chemical properties of globular clusters as constraints. This model has many implications on the observational properties of young clusters. However, young globular clusters are only found in distant galaxies such as NGC 1275 (Holtzman et al. 1992), where they cannot presently be resolved. However, recent observations find that most stars in the Galaxy today form in clusters in which the timescale for star formation is quite short (Lada et al. 1991; Lada 1992). The central density of some young clusters are comparable to that of some globular clusters. In the Trapezium cluster, all the stars appear to have ages $<10^{6} \mathrm{yr}$ (Prosser et al. 1994) which is $\sim 2 \tau_{d c}$. These timescales are consistent with the above model, in which star formation proceeds through a sequence of initial gas fragmentation, coagulation, protostellar collapse, and the clearing of residual gas. (There are exceptions such as IC 348, where star formation has persisted for many $\tau_{d c}$, Lada \& Lada, 1995). It is therefore of value to compare the implications of our model with the dynamical properties of nearby sites of ongoing star formation, to provide both supporting evidence and constraints upon the above picture of star formation.

Molecular clouds are clumpy on all scales (Scalo 1985; Zinnecker et al. 1993). Complex cloud substructure may also be inferred from the large 
dispersion, over a small field, in the observed extinction of the stars in the background cluster IC 5146 (Lada et al. 1994). (The extinction is equivalent to the surface density of the intervening clouds). These observations suggest that fragmentation occurs in the clouds prior to the gravitational collapse of individual protostellar cores as we have postulated above.

Magnetic fields, neglected in our cluster and star formation scenario, are observed to be important in regulating the structure of star forming regions today. In these regions, the velocity dispersions, which are correlated with the length scale of the substructures (Larson 1981), are often larger than the sound speed inferred from the transition temperature, but are comparable to the Alfvén speed (Heiles et al. 1993; Caselli \& Myers 1995). In some regions, the dispersive motion of the clouds may be regulated by the interstellar magnetic fields. There are also, however, magnetic supercritical regions, where the magnetic field can no longer balance gravity. These are the regions where massive stars and small clusters are formed. The lack of polarization in the densest cores of molecular clouds suggest that magnetic fields are excluded from these regions (Goodman et al. 1995). The decoupling of the field from the protostellar clouds is equivalent to the loss of thermal support during a cooling instability, and may also lead to complex substructures (Terquem \& Lin in preparation).

The mass functions of dark cloudlets in star forming complexes such as Ophiuchus, Taurus, Orion (Scalo 1985), and L1630 (Lada et al. 1991) have very similar power-law distributions, and are considerably flatter than that of the stellar IMF. The extrapolated collisional timescale for the small cloudlets is comparable to a few local dynamical timescales, and so their size distribution could arise naturally from a collisional equilibrium. The relatively flat mass spectrum is then consistent with that obtained from numerical simulations of the coagulation processes among protostellar cores (Murray \& Lin 1996), and implies that the physical cross section determines the merger rate among the cloudlets. In contrast, gravitational focusing is more important for the capture of residual cloudlets by collapsed stellar cores, leading to a steeper stellar IMF.

Shortly after the formation of protostellar cores, they are surrounded by the residual gas and appear as embedded sources. IR observations indicate that the embedded sources are strongly clustered (Greene et. al. 1994). These clusters are much more centrally condensed than the host cloud complex. Furthermore, the brightest embedded sources are usually found at the center of the clusters (Lada 1992). The luminosity segregation is consistent with the concept that protostellar cores form through dissipative mergers of small cloudlets, such that the most massive stars preferentially form in regions of high density where the collision frequency is greatest.

Finally, in older star forming regions, the young stellar objects emerge 
as T Tauri stars. In the Orion Nebula, T Tauri stars also appear to be clustered. The higher luminosity T Tauri stars are more centrally condensed than those with low luminosities (Prosser et al. 1994). In these regions, there is not sufficient time for post-formation dynamical evolution toward mass segregation. The observations are consistent with the more massive stars forming in dense, central regions. Since most of their kinetic energy is dissipated during the coagulation, these massive stars remain near PCC's center after the residual gas is cleared.

\section{References}

Aarseth, S.J., Lin, D.N.C., \& Papaloizou, J.C.B. 1988, ApJ, 324, 288

Binney, J. J. 1977, ApJ, 215, 483

Blumenthal, G. R., Faber, S. M., Primack, J. R., \& Rees, M. J. 1984, Nature, 311, 517

Bonner, W. B. 1956, MNRAS, 116, 356

Caselli, P. \& Myers, P.C. 1995, ApJ, 446, 665

Dalgarno, A., \& McCray, R. A. 1972, ARAA, 10, 375

Field, G. B. 1965, ApJ, 142, 531

Goodman, A.A., Jones, T.J., Lada, E.A., Myers, P.C. 1995, ApJ 448, 748

Greene, T.P., Wilking, B.A., André, P., Young, E., \& Lada, C.J. 1994, ApJ, 434, 614

Heiles, C., Goodman, A.A., \& McKee, C.F. 1993 in Protostars and planet III, eds. E. H. Levy \& J. I. Lumine (Tucson: Univ. Arizona Press), 279

Holtzman, J. A., et al. 1992, AJ, 103, 691

Hoyle, F. 1953, ApJ, 118, 513

Lada, C. J., Lada, E. A., Clemens, D. P., \& Bally, J. 1994, ApJ, 429, 694

Lada, C. J., Margulis, M., \& Dearborn, D. 1984, ApJ, 285, 141

Lada, E. A. 1992, ApJL, 1992, 393, L25

Lada, E. A., DePoy, D. L., Evans, N. J., \& Gatley, I. 1991, ApJ, 371, 171

Lada, E.A. \& Lada, C.J. 1995, AJ, 109, 1684

Larson, R. B. 1974, MNRAS, 169, 229 1981, MNRAS, 194, 809

Lin, D. N. C., \& Murray, S. D. 1992, ApJ, 394, 523

Lin, D. N. C., \& Pringle, J. E. 1987, ApJL, 320, L87

Low, C., \& Lynden-Bell, D. 1976, MNRAS, 176, 367

McKee, C. F., \& Cowie, L. L. 1977, ApJ, 215, 213

Murray, S. D., \& Lin, D. N. C. 1989, ApJ, 339, 933

- 1990, ApJ, 357, 105

- 1992, ApJ, 400, 265

- 1996, ApJ, 467, 728

Murray, S. D., White, S. D. M., Blondin, J. M., \& Lin, D. N. C. 1993, ApJ, 407, 588

Noriega-Crespo, A. Bodenheimer, P. Lin, D. Tenorio-Tagle, G. 1989, MNRAS, 237, 461

Prosser, C. F. et al. 1994, ApJ, 421, 517

Rees, M. J., \& Ostriker, J. P. 1977, MNRAS, 179, 541

Scalo, J.M. 1985, in Protostars and Planets II, eds. D.C. Black \& M. Matthews, (Univ. of Arizona Press), 201

Shu, F. 1977, ApJ, 214, 488

Stetson, P. B. \& Harris, W. E. 1988, AJ, 96, 909

Tenorio-Tagle, G. Bodenheimer, P. Lin, D. Noriega-Crespo, A. 1986, MNRAS, 221, 635

Wheeler, J. C., Sneden, C., \& Truran, J. W. 1989, ARAA, 27, 279

White, S. D. M., \& Rees, M. J. 1978, MNRAS, 183, 341

Zinnecker, H., McCaughrean, M., \& Wilking, B. A. 1993, in Protostars and Planets III, eds. E. Levy \& J. Lunine, (Univ. of Arizona Press), 429 